\title{
AROUND OPERATOR MONOTONE FUNCTIONS
}

\author{
MOHAMMAD SAL MOSLEHIAN AND HAMED NAJAFI
}

\begin{abstract}
We show that the symmetrized product $A B+B A$ of two positive operators $A$ and $B$ is positive if and only if $f(A+B) \leq f(A)+f(B)$ for all non-negative operator monotone functions $f$ on $[0, \infty)$ and deduce an operator inequality. We also give a necessary and sufficient condition for that the composition $f \circ g$ of an operator convex function $f$ on $[0, \infty)$ and a non-negative operator monotone function $g$ on an interval $(a, b)$ is operator monotone and give some applications.
\end{abstract}

\section{INTRODUCTION}

Let $\mathbb{B}(\mathscr{H})$ denote the algebra of all bounded linear operators on a complex Hilbert space $(\mathscr{H},\langle\cdot, \cdot\rangle)$. An operator $A \in \mathbb{B}(\mathscr{H})$ is called positive if $\langle A x, x\rangle \geq 0$ holds for every $x \in \mathscr{H}$ and then we write $A \geq 0$. The set of all positive operators on $\mathscr{H}$ is denoted by $\mathbb{B}(\mathscr{H})_{+}$. For self-adjoint operators $A, B \in \mathbb{B}(\mathscr{H})$, we say $A \leq B$ if $B-A \geq 0$. The symmetrized product of two operators $A, B \in \mathbb{B}(\mathscr{H})$ is defined by $S(A, B)=$ $A B+B A$. In general, the symmetrized product of two operators $A, B \in \mathbb{B}(\mathscr{H})_{+}$is not positive. For example, if $A=\left(\begin{array}{ll}1 & 0 \\ 0 & 0\end{array}\right)$ and $B=\left(\begin{array}{ll}1 & 1 \\ 1 & 1\end{array}\right)$, then $A B+B A$ is not a positive operator. The operator $\frac{1}{2} S(A, B)$ is called the Jordan product of $A$ and $B$. Nicholson [11] and Strang [12] introduced some sufficient conditions for that the Jordan product of two positive matrices $A$ and $B$ is positive. Also Gustafson [7] showed that if $0 \leq m \leq A \leq M$ and $0 \leq n \leq B \leq N$, then

$$
m n-\frac{(M-m)(N-n)}{8} \leq \frac{1}{2} S(A, B) .
$$

Throughout the paper we assume all functions to be continuous. Let $f$ be a real-valued function defined on an interval $J$. If for each self-adjoint operators $A, B \in \mathbb{B}(\mathscr{H})$ with spectra in $J$,

- $A \leq B$ implies $f(A) \leq f(B)$, then $f$ is called operator monotone;

2010 Mathematics Subject Classification. Primary 47A63; Secondary 47B10, 47A30.

Key words and phrases. Operator monotone function; Jordan product; operator convex function; subadditivity; composition of functions. 
- $f(\lambda A+(1-\lambda) B) \leq \lambda f(A)+(1-\lambda) f(B)$ for all $\lambda \in[0,1]$, then $f$ is said to be operator convex;

- $f(A+B) \leq f(A)+f(B)$, then $f$ is called subadditive.

If $f$ is an operator monotone function on $[0, \infty)$, then $f$ can be represented as

$$
f(t)=f(0)+\beta t+\int_{0}^{\infty} \frac{\lambda t}{\lambda+t} d \mu(\lambda),
$$

where $\beta \geq 0$ and $\mu$ is a positive measure on $[0, \infty)$ and if $f$ is an operator convex function on $[0, \infty)$, then $f$ can be represented as

$$
f(t)=f(0)+\beta t+\gamma t^{2}+\int_{0}^{\infty} \frac{\lambda t^{2}}{\lambda+t} d \mu(\lambda)
$$

where $\gamma \geq 0, \beta=f_{+}^{\prime}(0)=\lim _{t \rightarrow 0^{+}} \frac{f(t)-f(0)}{t}$ and $\mu$ is a positive measure on $[0, \infty)$; see $[5$, Chapter V]. If $f$ is a non-negative operator monotone function on $[0, \infty)$, then the subadditivity of $f$ does not hold in general. Aujla and Bourin [2] showed that if $A, B \geq 0$ are matrices and $f:[0, \infty) \rightarrow[0, \infty)$ is a concave function, i.e. $-f$ is convex, then there exist unitaries $U, V$ such that

$$
f(A+B) \leq U f(A) U^{*}+V f(B) V^{*} .
$$

Regarding the subadditivity property, Ando and Zhan [1] proved that $\||f(A+B) \|| \leq$ $\||| f(A)+f(B) \mid\|$ for all non-negative operator monotone functions $f$, all unitarily invariant norms ||$|\cdot|||$ and all matrices $A, B \geq 0$; see also [3]. Recall that a norm $\||\cdot|\|$ on the algebra of all $n \times n$ matrices is unitarily invariant if $\||X|\|=\|U X V \mid\|$ for all unitaries $U$ and $V$ and all matrices $X$.

Throughout this paper $\mathcal{M}(\mathscr{H})$ denotes the set of all $(A, B) \in \mathbb{B}(\mathscr{H})_{+} \times \mathbb{B}(\mathscr{H})_{+}$for which $f(A+B) \leq f(A)+f(B)$ for all non-negative operator monotone functions $f$ on $[0, \infty)$. We shall show that

$$
\mathcal{M}(\mathscr{H})=\left\{(A, B) \in \mathbb{B}(\mathscr{H})_{+} \times \mathbb{B}(\mathscr{H})_{+} \mid A B+B A \geq 0\right\} .
$$

We apply this result to present an operator inequality involving operator monotone functions analogue to that of Hansen [9]. We also give a necessary and sufficient condition for the composition $f \circ g$ of an operator convex function $f$ on $[0, \infty)$ and a nonnegative operator monotone function $g$ on an interval $(a, b)$ to be operator monotone and deduce some operator inequalities.

\section{The Results}

We start this section with one of our main results. 
Theorem 2.1. Let $A, B \in \mathbb{B}(\mathscr{H})_{+}$. Then $A B+B A$ is positive if and only if $f(A+B) \leq$ $f(A)+f(B)$ for all non-negative operator monotone functions $f$ on $[0, \infty)$.

Proof. Suppose that $f(A+B) \leq f(A)+f(B)$ for all non-negative operator monotone functions $f$ on $[0, \infty)$. Let $\lambda \in(0, \infty)$. The function $f_{\lambda}(t)=\frac{\lambda t}{\lambda+t}$ is operator monotone on $[0, \infty)$. Hence

$$
(A+B)(\lambda+A+B)^{-1} \leq A(\lambda+A)^{-1}+B(\lambda+B)^{-1}
$$

Put $X_{\lambda}=A(A+\lambda)^{-1}$ and $Y_{\lambda}=B(B+\lambda)^{-1}$. Inequality (2) is equivalent to

$$
\begin{aligned}
(\lambda+A+B)(A+B) \leq & (\lambda+A+B) X_{\lambda}(\lambda+A+B) \\
& +(\lambda+A+B) Y_{\lambda}(\lambda+A+B) \\
= & \lambda^{2} X_{\lambda}+2 \lambda A X_{\lambda}+\lambda B X_{\lambda}+\lambda X_{\lambda} B+A X_{\lambda} A+A X_{\lambda} B \\
& +B X_{\lambda} A+B X_{\lambda} B+\lambda^{2} Y_{\lambda}+2 \lambda B Y_{\lambda}+\lambda A Y_{\lambda} \\
& +\lambda Y_{\lambda} A+B Y_{\lambda} B+B Y_{\lambda} A+A Y_{\lambda} B+A Y_{\lambda} A .
\end{aligned}
$$

Since $B Y_{\lambda}=Y_{\lambda} B=B-\lambda Y_{\lambda}$ and $A X_{\lambda}=X_{\lambda} A=A-\lambda X_{\lambda}$ the above inequality is, in turn, equivalent to

$$
\begin{aligned}
\lambda(A+B)+A^{2}+B^{2}+A B+B A \leq & \lambda(A+B)+A^{2}+B^{2} \\
& +2(A B+B A)+B X_{\lambda} B+A Y_{\lambda} A
\end{aligned}
$$

or

$$
A B+B A+B X_{\lambda} B+A Y_{\lambda} A \geq 0
$$

Letting $\lambda \rightarrow \infty$ we get $B X_{\lambda} B+A Y_{\lambda} A \rightarrow 0$, whence $A B+B A \geq 0$.

Conversely, assume that $A B+B A \geq 0$. Since inequality (3) holds for each $\lambda \in \mathbb{R}_{+}$, we obtain $f_{\lambda}(A+B) \leq f_{\lambda}(A)+f_{\lambda}(B)$. Now, if $f$ be a non-negative operator monotone function on $[0, \infty)$, then $f$ can be represented on $[0, \infty)$ by

$$
f(t)=f(0)+\beta t+\int_{0}^{\infty} f_{\lambda}(t) d \mu(\lambda)
$$


where $\beta \geq 0$ and $\mu$ is a positive measure on $[0, \infty)$. Since $f(0) \geq 0$, without loss of generality, we can assume that $f(t)=\int_{0}^{\infty} f_{\lambda}(t) d \mu(\lambda)$. Therefore

$$
\begin{aligned}
f(A+B) & =\int_{0}^{\infty} f_{\lambda}(A+B) d \mu(\lambda) \\
& \leq \int_{0}^{\infty}\left(f_{\lambda}(A)+f_{\lambda}(B)\right) d \mu(\lambda) \\
& =\int_{0}^{\infty} f_{\lambda}(A) d \mu(\lambda)+\int_{0}^{\infty} f_{\lambda}(B) d \mu(\lambda) \\
& =f(A)+f(B) .
\end{aligned}
$$

If $A, B \in \mathbb{B}(\mathscr{H})_{+}$commute, then $A B=\left(A^{1 / 2} B^{1 / 2}\right)^{2} \geq 0$. The above theorem therefore shows that $f(A+B) \leq f(A)+f(B)$ for any non-negative operator monotone function $f$ on $[0, \infty)$. In particular, for each $n \in \mathbb{N}$ we have $f(n A) \leq n f(A)$.

Corollary 2.2. Let $0 \leq m \leq A \leq M, 0 \leq n \leq B \leq N$ and $f$ be a non-negative operator monotone function on $[0, \infty)$. If $(M-m)(N-n) \leq 8 m n$, then

$$
f(A+B) \leq f(A)+f(B) .
$$

Proof. Use inequality (1).

Corollary 2.3. Let $0 \leq p \leq \frac{1}{2}$ and $f$ be a non-negative operator monotone function on $[0, \infty)$. Then for positive operators $A$ and $B$ with $A \leq B$ it holds that

$$
f\left(B^{p}\right) \leq f\left(\frac{B^{p}+A^{p}}{2}\right)+f\left(\frac{B^{p}-A^{p}}{2}\right) .
$$

Proof. Assume that $S_{1}=\frac{1}{2}\left(B^{p}+A^{p}\right)$ and $S_{2}=\frac{1}{2}\left(B^{p}-A^{p}\right)$. Clearly $S_{1} \geq 0$ and due to $f(t)=t^{p}$ is operator monotone [6, Theorem 1.8], $S_{2} \geq 0$. We have

$$
2\left(S_{1} S_{2}+S_{2} S_{1}\right)=\left(S_{1}+S_{2}\right)^{2}-\left(S_{1}-S_{2}\right)^{2}=B^{2 p}-A^{2 p} \geq 0,
$$

since $f(t)=t^{2 p}$ is also operator monotone. Therefore

$$
f\left(B^{p}\right)=f\left(S_{1}+S_{2}\right) \leq f\left(S_{1}\right)+f\left(S_{2}\right)=f\left(\frac{B^{p}+A^{p}}{2}\right)+f\left(\frac{B^{p}-A^{p}}{2}\right) .
$$

Let $m \leq M \leq(2 \sqrt{2}+1) m$. If $0 \leq m \leq A, B \leq M$, then inequality (1) ensures that $A B+B A$ is positive. From this we can introduce an inequality for non-negative operator monotone functions. We should notice that Hansen [9] proved that an operator monotone function $f$ on $[0, \infty)$ satisfies the inequality $C^{*} f(A) C \leq f\left(C^{*} A C\right)$ for all 
positive operators $A$ and all contractions $C$, i.e. operators of norm less than or equal to one.

Theorem 2.4. Let $f$ be a non-negative operator monotone function on $[0, \infty)$. Let $A$ and $A_{i}(1 \leq i \leq n)$ be positive operators with spectra in $[\lambda,(1+2 \sqrt{2}) \lambda]$ for some $\lambda \in \mathbb{R}_{+}$. Then

(i) For every isometry $C \in \mathbb{B}(\mathscr{H})$,

$$
f\left(C^{*} A C\right) \leq 2 C^{*} f\left(\frac{A}{2}\right) C .
$$

(ii) For operators $C_{i}(i=1, \cdots, n)$ with $\sum_{i=1}^{n} C_{i}^{*} C_{i}=I$,

$$
f\left(\sum_{i=1}^{n} C_{i}^{*} A_{i} C_{i}\right) \leq 2 \sum_{i=1}^{n} C_{i}^{*} f\left(\frac{A}{2}\right) C_{i} .
$$

Proof. (i) Put $X=\left(\begin{array}{cc}A & 0 \\ 0 & A\end{array}\right), D=\left(I-C C^{*}\right)^{\frac{1}{2}}, V=\left(\begin{array}{cc}C & -D \\ 0 & C^{*}\end{array}\right)$ and $U=$ $\left(\begin{array}{cc}C & D \\ 0 & -C^{*}\end{array}\right)$. It is easy to see that $U$ and $V$ are unitary operators. So $\operatorname{sp}\left(U^{*} X U\right)=$ $\operatorname{sp}\left(V^{*} X V\right)=\operatorname{sp}(X) \subseteq[\lambda,(1+2 \sqrt{2}) \lambda]$, where "sp" stands for spectrum. Thus

$$
\begin{aligned}
\left(\begin{array}{cc}
f\left(C^{*} A C\right) & 0 \\
0 & f\left(D A D+C A C^{*}\right)
\end{array}\right) & =f\left(\begin{array}{cc}
C^{*} A C & 0 \\
0 & D A D+C A C^{*}
\end{array}\right) \\
& =f\left(\frac{U^{*} X U+V^{*} X V}{2}\right) \\
& \leq f\left(U^{*} \frac{X}{2} U\right)+f\left(V^{*} \frac{X}{2} V\right) \\
& =U^{*} f\left(\frac{X}{2}\right) U+V^{*} f\left(\frac{X}{2}\right) V \\
& =2\left(\begin{array}{cc}
C^{*} f\left(\frac{A}{2}\right) C & D f\left(\frac{A}{2}\right) D+C f\left(\frac{A}{2}\right) C^{*}
\end{array}\right)
\end{aligned}
$$

whence $f\left(C^{*} A C\right) \leq 2 C^{*} f\left(\frac{A}{2}\right) C$.

(ii) Set

$$
\widetilde{C}=\left(\begin{array}{c}
C_{1} \\
\vdots \\
C_{n}
\end{array}\right), \quad \widetilde{A}=\left(\begin{array}{cccc}
A_{1} & & & 0 \\
& A_{2} & & \\
& & \ddots & \\
0 & & & A_{n}
\end{array}\right)
$$


Thus

$$
f\left(\sum_{i=1}^{n} C_{i}^{*} A_{i} C_{i}\right)=f\left(\widetilde{C}^{*} \widetilde{A} \widetilde{C}\right) \leq 2 \widetilde{C}^{*} f\left(\frac{\widetilde{A}}{2}\right) \widetilde{C}=2 \sum_{i=1}^{n} C_{i}^{*} f\left(\frac{A}{2}\right) C_{i} .
$$

Corollary 2.5. Let $\lambda \in \mathbb{R}_{+}$and $\left.w_{i} \in[\lambda,(1+2 \sqrt{2}) \lambda)\right](i=1, \cdots, n)$. Let $f$ be $a$ non-negative operator monotone function on $[0, \infty)$ and $A_{i}$ be positive operators such that $\sum_{i=1}^{n} A_{i}=I$. Then

$$
f\left(\sum_{i=1}^{n} w_{i} A_{i}\right) \leq 2 \sum_{i=1}^{n} f\left(\frac{w_{i}}{2}\right) A_{i}
$$

Proof. Put $C_{i}=A_{i}^{\frac{1}{2}}$ in part (ii) of Theorem 2.4.

Theorem 2.6. Let $A, B \in \mathbb{B}(\mathscr{H})_{+}$. Then $B^{2} \leq A^{2}$ if and only if for each operator convex function $f$ on $[0, \infty)$ with $f_{+}^{\prime}(0) \geq 0$ it holds that

$$
f(B) \leq f(A)
$$

Proof. Let $A, B \in \mathbb{B}(\mathscr{H})_{+}$. Suppose that for each operator convex function $f$ on $[0, \infty)$ with $f_{+}^{\prime}(0) \geq 0$ we have $f(B) \leq f(A)$. Due to $f(t)=t^{2}$ is operator convex on $[0, \infty)$ and $f_{+}^{\prime}(0) \geq 0$, we get $B^{2} \leq A^{2}$.

Next we show the converse. First assume that $A$ and $B$ are invertible positive operators such that $B^{2} \leq A^{2}$. Then $\lambda A^{-2}+A^{-1} \leq \lambda B^{-2}+B^{-1}$. This inequality is equivalent to $\left(\lambda B^{-2}+B^{-1}\right)^{-1} \leq\left(\lambda A^{-2}+A^{-1}\right)^{-1}$ and this is, in turn, equivalent to $B^{2}(B+\lambda)^{-1} \leq A^{2}(A+\lambda)^{-1}$. On the other hand $f_{\lambda}(t)=\frac{\lambda t^{2}}{\lambda+t}$ is operator convex on $[0, \infty)$, so $f_{\lambda}(B) \leq f_{\lambda}(A)$.

If $A$ and $B$ are positive and $(A-B, A+B) \in \mathcal{M}(\mathscr{H})$, then for each $\epsilon \geq 0$ let us set $A_{\epsilon}=A+\epsilon$ and $B_{\epsilon}=B+\epsilon$. Then $\left(A_{\epsilon}-B_{\epsilon}, A_{\epsilon}+B_{\epsilon}\right) \in \mathcal{M}(\mathscr{H})$. Hence

$$
f_{\lambda}(B+\epsilon) \leq f_{\lambda}(A+\epsilon) .
$$

Letting $\epsilon \rightarrow 0$ we get $f_{\lambda}(B) \leq f_{\lambda}(A)$.

Now suppose that $f$ is an operator convex function on $[0, \infty)$ with $f_{+}^{\prime}(0) \geq 0$. It is known that $f$ can be represented on $[0, \infty)$ by

$$
f(t)=f(0)+\beta t+\gamma t^{2}+\int_{0}^{\infty} f_{\lambda}(t) d \mu(\lambda),
$$

where $\gamma \geq 0, \beta=f_{+}^{\prime}(0)$ and $\mu$ is a positive measure on $[0, \infty)$; see [5, Chapter V]. If

$$
f(t)=\int_{0}^{\infty} f_{\lambda}(t) d \mu(\lambda)
$$


then due to $f_{\lambda}(B) \leq f_{\lambda}(A)$ for each $\lambda \in \mathbb{R}_{+}$we have

$$
f(B) \leq f(A)
$$

Since $\gamma, \beta \geq 0$ and $B^{2} \leq A^{2}$ the validity of $f(B) \leq f(A)$ is deduced in the general case when $f$ is given by (4).

Remark 2.7. It follows from the identity

$$
(A-B)(A+B)+(A+B)(A-B)=2\left(A^{2}-B^{2}\right)
$$

that $B^{2} \leq A^{2}$ if and only if $(A-B, A+B) \in \mathcal{M}(\mathscr{H})$.

We need the Löwner Theorem for establishing our next main result.

Theorem 2.8. [10, Löwner Theorem] A function $g$ defined on $(a, b)$ is operator monotone if and only if it is analytic in $(a, b)$, can analytically be continued to the whole upper half-plane $\{z \in \mathbb{C}: \operatorname{Im}(z)>0\}$ and represents there an analytic function whose imaginary part is non-negative.

Theorem 2.9. Let $g$ be a non-negative operator monotone function on an interval $(a, b)$. Let $g(z)=u(z)+i v(z)$ be its analytic continuation to the upper half-plane. Then for each operator convex function $f$ on $[0, \infty)$ with $f_{+}^{\prime}(0) \geq 0, f \circ g$ is operator monotone on $(a, b)$ if and only if $u(z) \geq 0$ on the upper half-plane.

Proof. Assume that $u(z) \geq 0$. By the Löwner Theorem 2.8, $v(z) \geq 0$ on the upper halfplane. One then sees that $g$ maps the upper half-plane into the first quadrant of plane. Hence $g^{2}$ maps the upper half-plane into itself. Utilizing again the Löwner Theorem we conclude that $g^{2}$ is operator monotone. Assume that $A$ and $B$ are self-adjoint operators with spectra in $(a, b)$ and $B \leq A$. It follows from $(g(A)-g(B))(g(A)+g(B))+(g(A)+$ $g(B))(g(A)-g(B))=2\left(g(A)^{2}-g(B)^{2}\right)$ that $(g(A)-g(B), g(A)+g(B)) \in \mathcal{M}(\mathscr{H})$. Now Theorem 2.6 implies that for each operator convex function $f$ on $[0, \infty)$ with $f_{+}^{\prime}(0) \geq 0$ the function $f \circ g$ is operator monotone on $(a, b)$.

Conversely, assume that there would be a complex number $z_{0}$ with $\operatorname{Im} z_{0}>0$ such that $u\left(z_{0}\right)<0$. Because $g$ is operator monotone, $v\left(z_{0}\right) \geq 0$. Hence $\frac{\pi}{2}<\operatorname{Arg}\left(g\left(z_{0}\right)\right)<\pi$. Therefore $\pi<\operatorname{Arg}\left(g^{2}\left(z_{0}\right)\right)<2 \pi$. So that $\operatorname{Im} g^{2}\left(z_{0}\right)<0$. Using the Löwner Theorem $2.8, g^{2}$ is not operator monotone on $(a, b)$, which is a contradiction, since $f(x)=x^{2}$ is operator convex on $(0, \infty)$.

Remark 2.10. If a non-negative operator monotone function $g$ on an interval $(a, b)$ is not a zero constant function and $u(z) \geq 0$ on the domain of $g$, then $u(z)>0$ on this domain. To see this note that: 
(i) If for some $t_{0} \in(a, b)$ we have $g\left(t_{0}\right)=0$, then there exists $R>0$ such that $g$ can be represented as $g(t)=\sum_{n=1}^{\infty} \frac{g^{(n)}\left(t_{0}\right)}{n !}\left(t-t_{0}\right)^{n}$ for all $t \in\left(t_{0}-R, t_{0}+R\right)$ [4, p. 63]. Since $g$ is a non-negative monotone function, $g(t)=0$ for all $t \leq t_{0}$. Hence $g^{(n)}\left(t_{0}\right)=0$ for all $n$, so $g$ is zero on the neighborhood $\left(t_{0}-R, t_{0}+R\right)$ of $t_{0}$. Thus $\{t: g(t)=0\}$ is clopen. Hence $g$ is zero on $(a, b)$ contradicting the assumption above. Thus $g(t)>0$ on $(a, b)$. (ii) If $g \neq 0$ is a constant function, then clearly $u(z)>0$.

(iii) If $g$ is not a constant function, then by the open mapping theorem for non-constant analytic functions, $u$ maps the upper half-plane into $\{z: \operatorname{Im} z>0 \& u(z)>0\}$.

Corollary 2.11. Let $0 \leq p \leq \frac{1}{2}$ and let $f$ be an operator convex function on $[0, \infty)$ with $f_{+}^{\prime}(0) \geq 0$. If $B \leq A$, then

$$
f\left(B^{p}\right) \leq f\left(A^{p}\right)
$$

Proof. Since for $0 \leq p \leq \frac{1}{2}$ the function $g(t)=t^{p}$ is non-negative operator monotone and $g$ takes the upper half-plane into the first quarter of plane, by Theorem 2.9 we get $f \circ g$ is operator monotone on $[0, \infty)$.

Corollary 2.12. Let $0 \leq p \leq \frac{1}{2}$ and $f$ be a non-negative operator monotone function on $[0, \infty)$. Then for positive operators $A$ and $B$ with $B \leq A$,

(i) $B^{p} f\left(B^{p}\right) \leq A^{p} f\left(A^{p}\right)$;

(ii) If $f$ is strictly positive on $(0, \infty)$ and $A, B$ are invertible, then $A^{p-1} f\left(A^{p}\right) \leq$ $B^{p-1} f\left(B^{p}\right)$.

Proof. (i) Due to $f$ is operator monotone on $[0, \infty)$, by [8, Theorem 2.4], the function $g(t)=t f(t)$ is operator convex on $[0, \infty)$, hence $g_{+}^{\prime}(0)=f(0) \geq 0$. Corollary 2.11 then yields

$$
B^{p} f\left(B^{p}\right) \leq A^{p} f\left(A^{p}\right) .
$$

(ii) By part (i), $h(t)=t^{p} f\left(t^{p}\right)$ is operator monotone on $(0, \infty)$. By [8, Corollary 2.6]), $t h(t)^{-1}$ is operator monotone, hence

$$
B^{1-p} f\left(B^{p}\right)^{-1} \leq A^{1-p} f\left(A^{p}\right)^{-1}
$$

Therefore

$$
A^{p-1} f\left(A^{p}\right) \leq B^{p-1} f\left(B^{p}\right)
$$

Acknowledgment. The authors would like to sincerely thank the anonymous referee for useful comments and suggestions. 


\section{REFERENCES}

1. T. Ando and X. Zhan, Norm inequalities related to operator monotone functions, Math. Ann. 315 (1999), 771-780.

2. J.S. Aujla and J.C. Bourin, Eigenvalue inequalities for convex and log-convex functions, Linear Algebr Appl. 424 (2007) 25-35.

3. J.S. Aujla and F. Silva, Weak majorization inequalities and convex functions, Linear Algebra Appl. 369 (2003), 217-233.

4. J. Bendat and S. Sherman, Monotone and convex operator functions, Trans. Amer. Math. Soc. 79 (1955), 58-71.

5. R. Bhatia, Matrix Analysis, Springer, New York, 1997.

6. T. Furuta, J. Mićić Hot, J. Pečarić and Y. Seo, Mond-Pečarić Method in Operator Inequalities, Element, Zagreb, 2005.

7. K. Gustafson, Interaction antieigenvalues, J. Math. Anal. Appl. 299 (2004), no. 1, 174-185.

8. F. Hansen and G. Pedersen, Jensen's inequality for operators and Löwner's theorem, Math. Ann. 258 (1981/82), no. 3, 229-241.

9. F. Hansen, An operator inequality, Math. Ann. 246 (1979/80), no. 3, 249-250.

10. K. Löwner, Uber monotone matrix funktionen, Math. Z. 38 (1934), 177-216.

11. D.W. Nicholson, Eigenvalue bounds for $A B+B A$, with $A, B$ positive definite matrices, Linear Algebra Appl. 24 (1979), 173-183.

12. W.G. Strang, Eigenvalues of Jordan products, Amer. Math. Monthly. 63 (1962), 37-40.

Department of Pure Mathematics, Center of Excellence in Analysis on Algebraic Structures (CEAAS), Ferdowsi University of Mashhad, P.O. Box 1159, Mashhad 91775 , IRAN

E-mail address: moslehian@ferdowsi.um.ac.ir and moslehian@member.ams.org

Department of Pure Mathematics, Ferdowsi University of Mashiad, P.O. Box 1159, MAShHAd 91775, Iran

E-mail address: hamednajafi20@gmail.com 